\title{
MASS TRANSFER RATES IN ALGOL BINARIES DEDUCED FROM THEIR PERIOD CHANGES
}

\author{
D. S. HALL and S. G. NEFF \\ Dyer Observatory, Vanderbilt University, Nashville, Tenn., U.S.A.
}

\begin{abstract}
Average mass transfer rates in 23 Algol binaries are derived by analyzing their observed period changes within the framework of the Biermann-Hall period change model. These are compared with thermal mass loss rates computed from the dimensions which the cooler (mass-losing) component had at the onset of mass outflow. The agreement is quite good and confirms a claim made earlier that the subgiant components in Algol binaries are losing mass at a rapid (thermal) rate, not at a slow (nuclear) rate.
\end{abstract}

\section{Introduction}

In this paper mass transfer rates in Algol binaries are derived by analyzing their period changes within the framework of the Biermann-Hall period change model (Biermann and Hall, 1973; Hall, 1975 = Paper I). The purpose is to check the preliminary finding made in Paper I that in all Algols the cooler component (the subgiant which is filling its Roche lobe) seems to be losing mass at the thermal (rapid) rate, not at the nuclear (slow) rate as has been supposed heretofore in the mass transfer theory for post-main-sequence evolution of close binaries.

In Paper I a detailed analysis of the period changes observed for U Cep resulted in an average mass transfer rate of $\geqslant 7 \times 10^{-6} M_{\odot} \mathrm{yr}^{-1}$. It was gratifying to find this in very good agreement with the rapid rate of $10^{-5} M_{\odot} \mathrm{yr}^{-1}$ which had been calculated for U Cep with case A and case B mass transfer models (Plavec, 1973). What was surprising was the implication, based on consideration of representative values for period changes and absolute dimensions, that all Algols are presently undergoing rapid mass transfer.

In this paper $\mathrm{O}-\mathrm{C}$ curves for 23 Algol binaries are analyzed in order to derive mass transfer rates using equations given in Paper I, realistic absolute dimensions are derived with the technique of Hall (1974), and then the observed average mass transfer rate is compared with thermal mass loss rates computed using Equation (11) of Paczynski (1971). The 23 binaries are TW And, Y Cam, R CMa, RZ Cas, U Cep, RS Cep, SW Cyg, VW Cyg, WW Cyg, W Del, TW Dra, RX Gem, Y Leo, RW Mon, AQ Peg, $\beta$ Per, RW Per, ST Per, Y Psc, USge, RW Tau, XTri, and TX UMa. Throughout most of this paper the terminology is that used in Paper I.

\section{Mass Transfer Rates Deduced from Period Changes}

The best $\mathrm{O}-\mathrm{C}$ curves available were collected for each of the 23 binaries and in a few cases updated with recent well-determined times of minimum. Then epochs of sudden period decrease were identified by inspection. According to the Biermann-Hall model these are epochs when a dynamical instability in the outer layers of the cooler star causes a sudden outflow of mass. The subsequent gradual period increase following each sudden decrease, called $\Delta_{2} P$ in Paper I, was measured graphically. Then the time intervals separating the epochs of period decrease, $\Delta t$, were noted. 
According to Paper $I$ the equations relating period decrease $\Delta_{1} P$ and period increase $\Delta_{2} P$ with the amount of mass lost from the cooler star, $\Delta M_{c}$, are

$$
\frac{\Delta_{1} P}{P}=3\left[\frac{M}{M_{c}}-\frac{M}{M_{h}}-\left(\frac{M}{M_{c}}\right)\left(\frac{M}{M_{h}}\right)^{1 / 2} r_{r}^{1 / 2}\right] \frac{\Delta M_{c}}{M}
$$

and

$$
\frac{\Delta_{2} P}{P}=3\left[\left(\frac{M}{M_{c}}\right)\left(\frac{M}{M_{h}}\right)^{1 / 2} r_{r}^{1 / 2}\right] \frac{\Delta M_{c}}{M} .
$$

Both contain the parameter $r_{r}$, the relative radius of the ring, i.e., the radius of gyration of the transferred material which lets angular momentum be stored temporarily as rotation in or around the hotter star. Since some mass is lost from the binary system in some cases, one cannot put an upper limit on $r_{r}$ in Equation (1). But since the subsequent period increase results from assimilation of mass by the hotter star one can say that $r_{r}$ in Equation (2) will not exceed the radius of the Roche lobe of the hotter star. That is why Equation (2), rather than Equation (1), must be used to compute $\Delta M_{c}$. It should be noted that Equation (2) can measure only the mass transferred to and assimilated by the hotter star, not necessarily all the mass lost by the cooler star.

It can be argued that the Roche lobe of the hotter star is too generous an upper limit for $r_{r}$ in Equation (2). The amount of material transferred during each sudden period decrease is around $10^{-5}$ or $10^{-6} M_{\odot}$. If this much mass were assumed to reside in a large cylindrical disk surrounding the hotter star within its Roche lobe during the $\sim 25 \mathrm{yr}$ of gradual period increase, then its average density would be large enough or larger than that necessary to produce an optical depth of unity in the continuum. In this case the hotter star would appear effectively as large as its disk, as one sees in $\beta$ Lyr (Wilson, 1974). If the mass resides closer to the limb of the hotter star, then the disk would be even denser. Thus one cannot escape the conclusion that the bulk of the transferred mass resides no farther out than the observed radius of the hotter star itself, i.e., the radius determined by solution of broad band light curves. There is another way of seeing this. Batten (1970) shows that typical densities of observed circumstellar disks are around $10^{12}$ particles $\mathrm{cm}^{-3}$; this implies a total mass of only about $10^{-10} M_{\odot}$, an insignificant fraction of the $10^{-5}$ or $10^{-6} M_{\odot}$ which comes over in each sudden transfer. Thus the observed radius of the hotter star is used in this contribution to approximate $r_{r}$ in Equation (2).

To determine the average mass transfer rate one simply divides the average $\Delta M_{c}$ by the average $\Delta t$. The resulting rates range between $\left\langle\Delta M_{c} \mid \Delta t\right\rangle=1.4 \times 10^{-8} M_{\odot} \mathrm{yr}^{-1}$ for $\mathrm{R} \mathrm{CMa}$ and $7.8 \times 10^{-6} M_{\odot} \mathrm{yr}^{-1}$ for UCep. Since the observed period increases and the intervals between decreases are not equal for any given binary, there is an uncertainty in each value of $\left\langle\Delta M_{c} \mid \Delta t\right\rangle$. This uncertainty, given simply by the standard deviation of the mean, averages around $\pm 60 \%$.

\section{Absolute Dimensions}

For use in the next section absolute dimensions are needed for the two components of the 22 binaries. Realistic values were computed by the procedure of Hall (1974). This involved selecting for each binary the best available light curve solution and the spectral 
type of the hotter component, and then using the effective temperature scale of Morton and Adams (1968) and the mass-luminosity relation of McCluskey and Kondo (1972).

\section{The Thermal Mass Loss Rate}

In the past it has been thought that the thermal (rapid) phase of mass loss comes to an end because the Roche lobe of the mass-losing star begins expanding soon after the initial mass ratio is reversed. But, as shown in Paper $I$, the Roche lobe is in fact shrinking as mass loss proceeds. Also it has been thought that the cooler components in the Algols are not subject to dynamical instability because it has been thought that the mass-losing component becomes convective at a time when expansion of its Roche lobe can forestall this instability. But the present shrinking of the Roche lobe shows why one should actually expect dynamical instabilities. Each dynamical mass loss stops after a finite although large amount of mass is lost. The nature of the dynamical instability is not yet understood in enough detail to tell us what determines the amount of mass lost each time. But Paper I argued that it is reasonable to suppose that, since the interval between dynamical outbursts is governed by the thermal relaxation time, the observed interval will be just that interval needed to make the average mass loss rate equal the thermal mass loss rate for the cooler star. This is the motivation in this contribution for comparing $\left\langle\Delta M_{c} / \Delta t\right\rangle$ with calculated thermal rates.

Paczynski (1971) showed that the maximum (thermal) rate of mass loss computed for 24 model binaries agreed, within a factor of three in most cases, with the formula

$$
\left(\frac{\mathrm{d} M}{\mathrm{~d} t}\right)_{\text {calc }}=3.2 \times 10^{-8} \frac{\left(R / R_{\odot}\right)\left(L / L_{\odot}\right)}{(M / M)_{\odot}} M_{\odot} \mathrm{yr}^{-1}
$$

Here $R, L$, and $M$ are the radius, bolometric luminosity, and mass of the mass-losing component at the onset of mass outflow. Thus it is convenient to use this formula to approximate expected thermal mass loss rates.

One must turn the clock back to find the dimensions of each binary just before the mass-losing component first made contact with its Roche lobe. To do this an initial mass ratio of 1.2 (see Paper I) is assumed, and conservation of total mass and orbital angular momentum is assumed during transfer. This lets the original semi-major axis $a^{0}$ come from the present semi-major axis $a^{p}$ with the formula

$$
\frac{a^{0}}{a^{p}}=\left[\frac{M_{1}^{p} M_{2}^{p}}{M_{1}^{0} M_{2}^{0}}\right]^{2}
$$

of Plavec (1970, Equation (7)), where subscripts 1 and 2 refer to the original primary and secondary components, respectively. Then the radius of the primary comes from the known relation between Roche lobe dimensions and mass ratio, and the luminosity comes from the mass-luminosity relation.

\section{The Comparison}

Before the comparison is made, it must be explained that the $\left\langle\Delta M_{c} / \Delta t\right\rangle$ values are expected to be systematically smaller than the real mass loss rate, and the $(\mathrm{d} M / \mathrm{d} t)_{\text {calc }}$ values probably underestimate the thermal mass loss rate. 
Mass loss rates deduced from the O-C curves are probably lower limits for a variety of reasons. First, because scatter in most $\mathrm{O}-\mathrm{C}$ curves makes it unclear just how many abrupt period changes really occurred, the analysis in this contribution has been conservative and only period decreases which were reasonably certain have been identified. Thus it may be that the measured $\Delta t$ values are too long. Second, experience with U Cep (Paper I), X Tri (Mallama, 1975), and RZ Cas (Hall et al., 1976) has shown that closely spaced period decreases tend to have relatively larger period increases in between. Thus it may be that some relatively large values of $\Delta_{2} P$ tend to be missed. Third, Equation (2) can measure only the mass assimilated by the hotter star, not any mass which might be lost from the system. Fourth, as explained in Paper I, it is probable that some angular momentum is being stored semi-permanently in at least some systems, in which case $\Delta_{2} P$ would underestimate $\Delta M_{c}$ to that extent. Thus except for U Cep and RZ Cas, for which well-defined O-C curves were represented very well, the derived values of $\left\langle\Delta M_{c} / \Delta t\right\rangle$ might underestimate the true rate of mass loss from the cooler star by something like a factor 3 to 5 on the average; but this is a very crude guess.

Thermal mass loss rates approximated with Equation (3) are going to be too low because the calculated initial values of $R, L$, and $M$ assumed conservation of mass and orbital angular momentum during transfer, which is almost certainly not right. If one guesses that about $\frac{1}{4}$ of the transferred mass is lost from the system and that non-conservation of orbital angular momentum has caused the present semi-major axis to be smaller by a factor 2 than it would have been with conservative transfer, then the Equation (3) values would be too small by a factor 2.5. This can be checked for U Cep and U Sge, for which model calculations (Plavec, 1973) give $10^{-5} M_{\odot} \mathrm{yr}^{-1}$ and $3 \times 10^{-6} M_{\odot} \mathrm{yr}^{-1}$, respectively, but for which use of Equation (3) gives $3.34 \times 10^{-6} M_{\odot} \mathrm{yr}^{-1}$ and $1.51 \times 10^{-6} M_{\odot} \mathrm{yr}^{-1}$, respectively. Since for these two the discrepancies are 3 and 2, one can have some confidence in the estimate 2.5 .

Since both $\left\langle\Delta M_{\mathrm{c}} / \Delta t\right\rangle$ and $(\mathrm{d} M / \mathrm{d} t)_{\text {calc }}$ are systematically low by roughly comparable factors (3-5 versus 2.5), it was decided to look at the correlation directly, simply plotting $\log \left\langle\Delta M_{c} \mid \Delta t\right\rangle$ versus $\log (\mathrm{d} M / \mathrm{d} t)_{\text {calc }}$. The result, shown in Figure 1 , indicates surprisingly good agreement. Over a range of nearly three powers of ten, the average point misses the line of perfect correlation by only a factor two $(0.3$ in the $\log )$ and the worst point misses by only a factor five ( 0.7 in the $\log )$.

The agreement is quite good considering the uncertainties entering at various stages. The statistical uncertainty of each $\left\langle\Delta M_{c} / \Delta t\right\rangle$ value is around $\pm 60 \%( \pm 0.2$ in the log). The $(\mathrm{d} M / \mathrm{d} t)_{\text {calc }}$ values assumed an average value for the initial mass ratio, used an approximate mass-luminosity relation, and were based on Equation (3), which Paczynski showed is itself only an approximation. And there were the various systematic errors possibly affecting both $\left\langle\Delta M_{c} / \Delta t\right\rangle$ and $(\mathrm{d} M / \mathrm{d} t)_{\text {calc }}$.

It is concluded that analysis of O-C curves within the framework of the Biermann-Hall model confirms the initial suspicion that the subgiant components in Algol binaries are losing mass at a rapid (thermal) rate.

\section{Acknowledgement}

Support from the Vanderbilt University Research Council is acknowledged. 


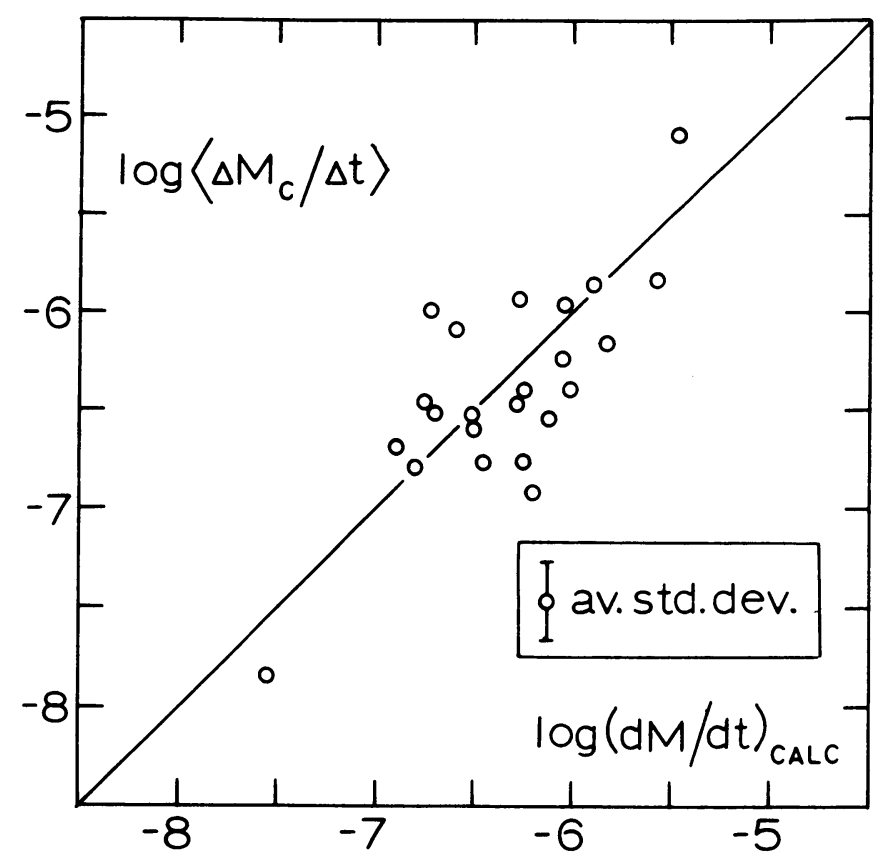

Fig. 1. The average mass transfer rate deduced from period changes, $\left\langle\Delta M_{c} / \Delta t\right\rangle$, plotted versus the calculated thermal mass loss rate $(\mathrm{d} M / \mathrm{d} t)_{\mathrm{calc}}$. The units of both are $M_{\odot} \mathrm{yr}^{-1}$. The error bar illustrates the average uncertainty of the $\left.\left\langle\Delta M_{c}\right\rangle \Delta t\right\rangle$ values. The agreement with the line of correlation is quite good, considering that most of the points are affected by some additional uncertainty.

\section{References}

Batten, A. H.: 1970, Publ. Astron. Soc. Pacific 82, 574.

Biermann, P. and Hall, D. S.: 1973, Astron. Astrophys. 27, 249.

Hall, D. S.: 1974, Acta Astron. 24, 215.

Hall, D. S.: 1975, Acta Astron. 25, 1 (Paper I).

Hall, D. S., Keel, B., and Neuhaus, G.: 1976, Acta Astron. 26, in press.

Mallama, A. D.: 1975, Acta Astron. 25, 205.

McCluskey, G. E. and Kondo, Y.: 1972, Astrophys. Space Sci. 17, 134.

Morton, D. C. and Adams, T. A.: 1968, Astrophys. J. 151, 611.

Paczynski, B.: 1971, Ann. Rev. Astron. Astrophys. 9, 183.

Plavec, M.: 1970, Publ. Astron. Soc. Pacific 82, 957.

Plavec, M.: 1973, IAU Symp. 51, 216.

Wilson, R. E.: 1974, Astrophys. J. 189, 319.

\section{DISCUSSION}

Paczynski: What happens to orbital angular momentum if the period stays constant?

Hall: There are two possibilities. Either some angular momentum remains stored as rotation deeper down in the target star and is not returned to the orbit, or else there is mass and angular momentum loss from the system. I think the second possibility is the case in these long-period Algol binaries in which the target star is small relative to the orbital semi-major axis, is not struck directly by the gas stream, and hence will have trouble assimilating a sudden transfer of $\sim 10^{-5} M_{\odot}$ on a dynamical 
time scale. I suspect a disk or shell of relatively high density forms temporarily and the resultant high gas pressure drives much of the transferred mass and angular momentum from the system.

Van den Heuvel: If you have, on the average, no period change at all, why could not we assume that, on the average, these systems do not have a net rate of mass transfer, i.e. as much mass is transferred forward as was transferred backwards.

Hall: Although your idea could work in principle, you will have serious trouble explaining why the hotter star, which is smaller than its Roche lobe, would return all of the mass. On the other hand, the Biermann-Hall model has mass loss only from the cooler star, which fill its Roche lobe, and hence is consistent with the way it is generally accepted that mass transfer proceeds in semi-detached binaries.

Wilson: If the rapid phase never cuts off, can you tell us why so many of these binaries are still around?

Hall: I am not sure there is a problem. We need to compare the number of main-sequence binaries available as suitable progenitors with the number of Algols observed and see if the ratio is consistent with the relative lifetime. I would claim that when this is done, there will be no problem; but to my knowledge this has not been done yet one way or the other.

Wilson: I suggest that a problem of terminology is involved here. The mass transfer rates you list are smaller than one normally associates with the rapid phase of mass transfer, so perhaps it would be better to say that "the slow phase is not as slow as you thought it was" rather than that there is no slow phase.

Hall: I was referring to the classical distinction (made in theoretical mass-transfer calculations) between an initial 'rapid' thermal mass loss phase followed by a 'slow' nuclear phase. Then, of course, these rather low-mass Algol binaries do have lower thermal mass-loss rates than the more massive Algol binaries like UCep. That is why you thought of them as slow. But the corresponding nuclear mass loss rates would be even slower, by a couple of orders of magnitude.

Ritter: Did your investigation include the very well observed system AS Eri (Popper)? A theoretical study by Refsdal, S., Roth, M. L., Weigert, A.: 1974, Astron. Astrophys. 36, 113, showed that during its foregoing evolution the system must have lost mass and angular momentum as well.

Hall: No it did not, but I do not recall now why it did not. I think it is because we were not able to locate a good $\mathrm{O}-\mathrm{C}$ curve to analyze. 